\title{
Gary D Hammer on the improvement of patient care in endocrine neoplasia
}

Gary D Hammer*,1

${ }^{1}$ Endocrine Oncology Program, Rogel Comprehensive Cancer Center, University of Michigan, Ann Arbor, MI 48105, USA

*Author for correspondence: ghammer@umich.edu

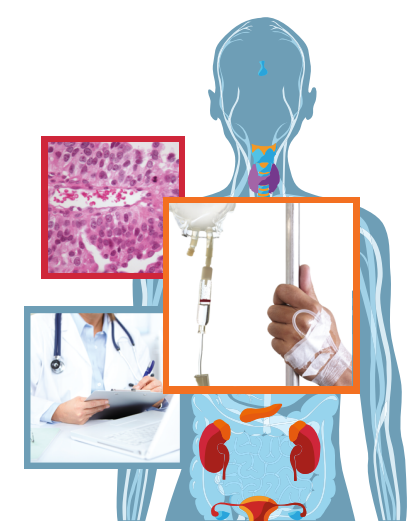

International Journal of Endocrine Oncology

Dr Gary D Hammer is the cofounder of Millendo Therapeutics (NASDAQ, MLND, MI, USA) and the founder of Vasaragen (MI, USA), two biotechnology companies focused on rare endocrine diseases. Hammer is also an employee of the University of Michigan.

First draft submitted: 2 December 2019; Accepted for publication: 2 December 2019; Published online: 23 December 2019

Gary D Hammer is the Director of the Endocrine Oncology Program at the University of Michigan Rogel Cancer Center (MI, USA). He is also the President-Elect of the Endocrine Society, the world's largest professional organization of endocrinologists and endocrine scientists. In addition, Hammer is the cofounder of Millendo Therapeutics (NASDAQ, MLND, MI, USA) and the founder of Vasaragen (MI, USA), two biotechnology companies focused on rare endocrine diseases.

Hammer's laboratory at the University of Michigan currently explores signaling and transcriptional control of stem cell lineages within the adrenal cortex and focuses on the development of novel therapeutics for adrenal neoplasia.

\section{How \& why was the Endocrine Neoplasia Program initiated at the University of Michigan?}

Our program at the University of Michigan Rogel Cancer Center is dedicated to the care of patients with endocrine neoplasias.

In 1999, when I came to the University of Michigan, I was particularly interested in merging my clinical and scientific interest in adrenal disease. The University of Michigan had a rich history of adrenal research and care of patients with adrenal diseases, dating back seven to eight decades. Some examples are:

- Metaiodobenzylguanidine was developed for the diagnosis and treatment of tumors of the adrenal medulla (pheochromocytomas);

- NP59, (iodocholesterol) was developed as an imaging agent to diagnose adrenal cortical tumors [1];

- Conn syndrome or primary aldosteronism was discovered by Jerome Conn (prior Division Chief of Endocrinology) in the 1950s [2].

A few years prior to my arrival, the University of Michigan had launched a fellowship program to formally train surgeons in endocrine surgery. Our cancer center had opened a few years earlier and due in part to the fundraising by Bo Schembechler (MI, USA), there was a chance to grow something larger, more comprehensive, in a true multidisciplinary manner that focused on endocrine neoplasia.

\section{Why is multidisciplinary care in endocrine oncology so important \& how did you develop a multidisciplinary team?}

We know that a multidisciplinary approach provides optimal care in many diseases. In oncology, the multidisciplinary clinic has been valued for some time, but is very hard to implement. At the University of Michigan Rogel Cancer Center, we have strived to build a clinic that embraces the model of multidisciplinary comprehensive care. It is difficult to build a program in a pre-existing environment of siloed care. To combat this, we (the nascent endocrine oncology 'team') set aside one day per week for multidisciplinary care. On that one day, only patients who required a multidisciplinary team would be seen. We focused on endocrine neoplasia because those tumors 
are routinely cared for by endocrinologists and endocrine surgeons. These patients often also require medical oncologists, nuclear medicine doctors, radiation oncologists, cancer geneticists, etc., and would see such specialists as necessary. As new faculty were recruited, it was made clear that every Tuesday, their only responsibility was to engage in the multidisciplinary care program. This strategy enabled us to fully build, brick-by-brick, a program where every doctor was available on that day to see patients. It sounds simple but was challenging because each faculty member was hired into a different department that had unique expectations and needs.

For our first 5 years, we focused on the patient experience. Despite the fact our program was embedded in a large university hospital system, we ensured that all records were obtained and evaluated before we saw the patients in the clinic. We implemented this process in thyroid cancer and adrenal neoplasia first, employing thyroid-focused endocrinologists, adrenal-focused endocrinologists, endocrine surgeons and one medical oncologist dedicated to endocrine oncology. Ancillary services were utilized as needed. Our workflow expanded with the engagement of a team including a specialized endocrine pathologist, adrenal radiologist, nuclear medicine physicians, radiation oncologist and ultimately cancer geneticist as well as database-tumor registry specialists/trial coordinators.

Accomplishing 'one-stop' shopping requires commitment from the practice group. Whether it is a university or a large health system, several pieces must fall into place and this can take years. Consider call centers, where you may have five or more different departments engaged. You may have calls coming into radiology, to oncology, to surgery and to endocrinology. How do you streamline the process, so that when a patient calls a number, it does not take ten different connections to reach the right program? We spent 2 years optimizing our call flow to ensure that the patient would get to us within two calls. Therefore, we could triage those needing true multidisciplinary care versus immediate referral to surgery, or alternatively tempered referral to our general endocrinology clinic.

Next, we focused on an expedient yet complete mechanism to receive all relevant information regarding each patient before their visit, which took another few years. After optimization, we are able to see any patient in the world within 10-14 days. Once in the clinic, we had to operationalize the visit and care plan involving multiple providers. The standard intake response of "we will get back to you in a couple of weeks when we have reviewed the scans" was not going to work. Particularly in the case of rare disease patients traveling from afar. Patients do not want to spend money on a flight, a hotel and a visit and to be told, "nice to meet you, we will talk to you in a couple of weeks." For this reason, we decided that everything would be reviewed prior to the patient's appointment. We would then see the patient and on the same day - a multidisciplinary board discussion would take place. We discuss every patient that needs multidisciplinary decision making and go back to see the patient after the board review in order to give them a codified plan before they boarded a plane to return home. Some of those patients will return for care and others we will aid in the coordination of care across the country. A program like this does not easily generate positive margins for a health system where relative value unit generation has become the metric of success. However, this program exemplifies the meaning of multidisciplinary care and how to provide quality, optimal patient-centered care for rare disease patients in the endocrine oncology space.

Now we see patients and coordinate care around the country, sometimes around the globe, for these rare diseases. For patients who were too sick to travel, we wanted to be able to provide them with recommendations for the ultra-rare adrenal cortical carcinoma. This is where telemedicine came in. We designed and implemented a Remote Second Opinion Program that involved the Endocrine Oncology Program members from the areas of endocrinology, oncology, radiology and pathology. We would not see the patients in person, but we would review the patient in the same way, except without a physical exam. All laboratory, radiologic and pathologic studies still needed to be reviewed before we brought the case to the board. This service foreshadowed the explosion of telemedicine in a variety of disciplines.

\section{How important is global engagement in program development?}

Our engagement with patient advocacy, both within our Michigan team and with our global collaborations, grew as social media platforms exploded and patients formed self-driven advocacy groups. The empowered patient voice began to be heard - by insurance companies, big Pharma and funding agencies like the NIH and the US FDA, not to mention Congress.

The challenge has been that for many rare diseases, most doctors have never seen a case of the disorder and require consultation. Patients with rare cancers are often disenfranchised because the healthcare system often does not allow patients with rare diseases to seek care with an expert. In our third-party payment system, 'out of network' consultation and referrals are discouraged or simply not allowed. We needed to find a way, in the US heath system 
and across the world, to care for patients who are just as needy and worthy of care as those with common diseases. Patients began advocating for themselves and each other to be able to receive care at institutions with expertise.

Our program expanded and began drawing patients from near and far. We soon realized that when a patient came to see us from across the country or across the ocean, it was unconscionable and impossible to spend only 30 min with them. These patients are often with us for 3-4 h receiving their comprehensive care plan.

The question is how do you provide that level of care? How can you accomplish this level of care more broadly - i.e., not only in a niche program like Endocrine Oncology at Michigan? Almost 10 years ago, one of our patients Rick Ally happened to be a senior advisor for then Senator Edward M Kennedy. Ally developed adrenal cancer while Senator Kennedy developed glioblastoma. I was asked to help write the rare cancer section of a new cancer bill, 'the 21st Century Access to Life-Saving Early Detection, Research and Treatment (ALERT) Act' [3], with my colleague Kavita Pate, who at that time was Deputy Staff Director of Health Education, Labor and Pensions Committee for Senator Kennedy and is now at Venture Partner, New Enterprise Associates (MA, USA). The section aimed to provide research and clinical support for those with rare cancers and to develop a program that provided access to Centers of Excellence for these cancers.

We had some roadblocks as we went around the country for 2 years trying to engage potential political and healthcare advocates. The biggest challenge was - and remains - that the word 'rare' is legislated. In the USA, it is legislated in the disease space by the Orphan Drug Act of 1983, which defines rare as a disease affecting fewer than 200,000 individuals per year [4]. That is a fine definition if your goal is to encumber as many uncommon diseases as possible to achieve some support for those patients. However, as a result of this definition every cancer is rare with the exception of 3-4 cancer types. Only two cancers - breast and lung - have an incidence of more than 200,000 individuals per year in the USA [5], meaning that cancer itself is a rare disease.

The goal of this particular program we were trying to implement was not to help all cancer, although all cancer needs to be cured, but to provide support for patients with what I will term orphan cancers for which there were no treatments. The number needed to be debated. Should it be 1000, 10,000, 20,000 or 40,000 per year? Regardless, we tried to get support for these ultra-rare diseases. We ultimately presented our work at a National Cancer Institute (MD, USA) conference where we discussed rare cancers: how to define them, how to advance science and how to develop effective treatments. The concept that grew out of the genomic revolution was that all cancer, in a sense, is rare. The cancer of each individual patient has unique genomic phenotypes that can be defined as rare. This coincided with the beginning of personalized medicine in oncology. The bill was announced and presented to the floor of the Congress but, ultimately was subsumed by healthcare reform.

\section{What role does patient advocacy play in research, program development \& clinical care?}

I am still engaged deeply in that space. In my upcoming role as the President of the Endocrine Society, I am deeply committed to two things:

- Empowering the voice of the patient in our professional societies and the power of the patient in advocacy;

- Supporting endocrine neoplasia research and care by advocating to Pharma, professional organizations and the governments of the world, to ultimately bring new effective therapies to these patients.

Recently, patient groups have taken center stage and the voice of the patient is becoming increasingly heard. We know that direct-to-patient marketing is rampant right now, but patients are taking notice and taking charge of their diseases. Patients are raising funds, starting their own foundations and finding cures for their own diseases; for example, look at the ice bucket challenge for amyotrophic lateral sclerosis. This challenge raised hundreds of millions of dollars for amyotrophic lateral sclerosis research and was $100 \%$ patient driven. Patients are more and more engaged. They are being asked to sit on the advisory boards of Pharma companies and funding agencies, in order to set priorities and review grant proposals.

I think that patient engagement and empowerment will remain critical over the next 10-20 years. The doctors and scientists who engage with these groups are going to be the ones who successfully navigate the landscape of both funding science and ultimately finding cures. Creating teams that involve patients, patient advocates, doctors and scientists, working together to advance the science into the clinical space is the only way that we are going to make a difference. I urge all of us in our various capacities to be open to such engagement, whether it is with our professional societies, with our patient advocacy groups, or on the floor of Congress. 
If you would like to hear more about patient advocacy [6] or Dr Hammer's TEDxUofM talk on vulnerability and presence in science and medicine [7] please follow the references mentioned in this article.

\section{Financial \& competing interests disclosure}

The authors have no relevant affiliations or financial involvement with any organization or entity with a financial interest in or financial conflict with the subject matter or materials discussed in the manuscript. This includes employment, consultancies, honoraria, stock ownership or options, expert testimony, grants or patents received orpending, or royalties.

No writing assistance was utilized in the production of this manuscript.

\section{Disclaimer}

The opinions expressed in this interview are those of the interviewee and do not necessarily reflect the views of Future Medicine Ltd (Future Science Ltd).

\section{Open access}

This work is licensed under the Attribution-NonCommercial-NoDerivatives 4.0 Unported License. To view a copyof this license, visit http://creativecommons.org/licenses/by-nc-nd/4.0/

\section{References}

1. University of Michigan Internal Medicine Research History (2019). www.medicine.umich.edu/dept/intmed/research/research-history

2. University of Michigan Internal Medicine Jerome W. Conn (2019). https://medicine.umich.edu/dept/intmed/divisions/metabolism-en docrinology-diabetes/about-us/jerome-w-conn-alumni-society/jerome-w-conn

3. S.717 (111th): 21st Century Cancer ALERT (Access to Life-Saving Early detection, Research and Treatment) Act (2009). www.govtrack.us/congress/bills/111/s717/text

4. Asbury CH. The Orphan Drug Act. The first 7 years. JAMA 265(7), 893-897 (1991).

5. Siegel RL, Miller KD, Jemal A. Cancer statistics 2019. CA Cancer J. Clin. 69(1), 7-34 (2019).

6. YouTube Adrenal Spencer Bell Legacy (2016). www.youtube.com/watch?v=CbwUxDfajRY\&feature=youtu.be

7. YouTube TedxUoM (2014). www.youtube.com/watch?v=mlkNhmBsFIs\&feature=youtu.be 